\title{
Horizontal Displacement Prediction Research of Deep Foundation Pit Based on the Least Square Support Vector Machine
}

\author{
Wei-Dong Li \\ College of Surveying and Prospecting Engineering, \\ Jilin Jianzhu University \\ Changchun, China
}

\author{
Meng-Hong Wu \\ College of Geoexploration Science and Technology, \\ Jilin University \\ Changchun, China
}

\author{
Nan Lin \\ College of Surveying and Prospecting Engineering, Jilin Jianzhu University \\ E-mail:1039283970@qq.com
}

\begin{abstract}
Using of the least square support vector machine to predict the horizontal displacement of deep foundation pit. According to the measured time series data of horizontal displacement of foundation pit, using the least square support vector machine (SVM) to set up the relation model of foundation pit horizontal displacement and time, taking the actual excavation monitoring data as learning and training samples and testing samples, the calculated results and the actual monitoring results were compared and analyzed. The results show that using the least squares support vector machine (SVM) to predict the horizontal displacement of foundation pit, which was with higher prediction accuracy, the method with prediction error is small, fast calculation, less data, etc., precision can satisfy the need of engineering. The method Confirmed that is an effective method to solve the problem of the foundation pit deformation prediction.
\end{abstract}

Keywords-Least square support vector machine; Deep foundation pit; Horizontal displacement; prediction.

\section{INTRODUCTION}

The rapid construction of urban high-rise buildings and the underground traffic network promote deep foundation pit engineering increasing in number and size, deep foundation pit in excavation process in the soil stress release will lead to pit deformation, the safety of foundation pit and surrounding buildings will have a serious impact, so it is particularly important to monitor and predict deformation of the deep foundation pit. Accurate prediction of deformation of deep foundation pit has been a hot research topic in recent years. Many scholars have conducted in-depth research and achieved fruitful results, Support vector machine technology are widely used in the results of its small sample training, support high dimensional feature space, fast convergence and other characteristics ${ }^{[1-3]}$. Due to the traditional prediction method has certain limitation, this paper apply the least square support vector machine model to predict the deep foundation pit horizontal displacement deformation based on the support vector machine principle, the model can effectively solve problems contain small sample, nonlinear, high dimension and local minima, etc.

\section{STUDY AREA (ENGINEERING SURVEY)}

Jilin Province People's hospital medical comprehensive building is located in the intersection of Hongqi Street and Xinyi Road in Changchun. It is in Jilin Province People's Hospital. The project is medical comprehensive building foundation pit; excavation depth is about 1.74 meters, excavation perimeter of 363.7 meters. In order to grasp the foundation deformation, to find adverse sinking phenomenon of construction timely, and to take measures to ensure the construction carried out smoothly. As the same time, the safety use of surrounding buildings and providing information for the future rational design are the reasons to monitor the construction of this project.

There are many inflection points in foundation pit, and the edge of each is unequal, we arrange 22 horizontal displacement observation points according to a certain distance, which distributed each corner and straight line segments of foundation pit. Each point masonry $300 * 300 * 300 \mathrm{~mm}$ (long $*$ wide $*$ high) concrete pier in the top of crown beam, and install spherical horizontal observation point mark in concrete pier, Schematic diagram of point plane as shown in Figure 1.

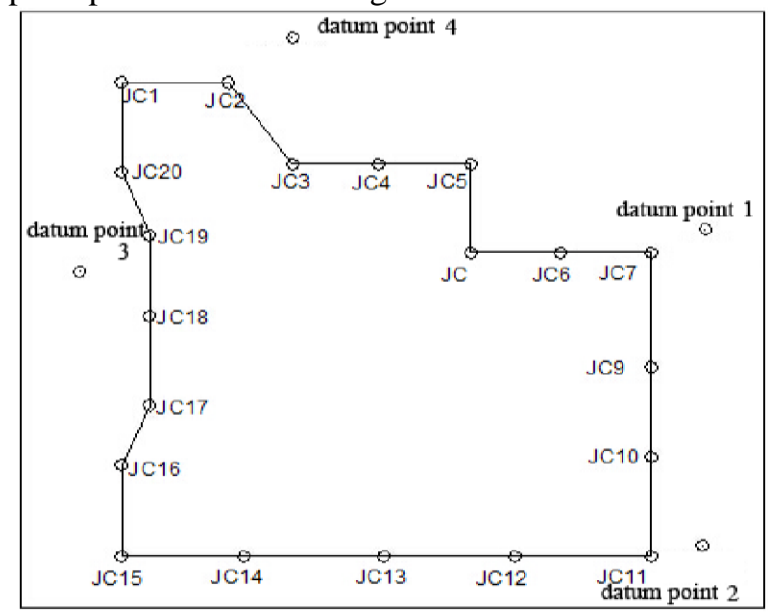

Figure 1. Layout plan of reference point and monitoring point 


\section{METHOD}

Support vector machine SVM is a machine learning algorithm based on statistical learning theory, the input vector from the original space is mapped to high dimensional feature space by selecting the corresponding nonlinear mapping function, using the rule of structure risk minimization, reducing upper bound of the generalization error of a model while minimizing the sample error, so as to enhance the generalization ability of the model. Compared with traditional machine learning algorithm, SVM has the advantages of high precision, fast computing speed characteristics.

For nonlinear separable samples, the dual form of SVM is [4-6]

$$
\max \sum_{i=1}^{n} \alpha_{i}-\frac{1}{2} \sum_{i, j=1}^{n} \alpha_{i} \alpha_{j} y_{i} y_{j} K\left(x_{i}, x_{j}\right)
$$

In the formula, $K\left(x_{i}, x_{j}\right)$ is a kernel function to use for carrying out nonlinear mapping, and the different forms of kernel function are chosen to represent the different nonlinear mapping, which lead the samples to map from the original space to the high dimensional feature space.

Least squares support vector machine (SVM) is an extension of a support vector machine (SVM), the least squares linear system replace the traditional support vector machine to solve the problem of pattern recognition, quadratic programming method is adopted to reduce the computation complexity and improve the solving speed, mainly reflected in: The empirical risk is changed from Once Party to the Two party, equality constraints instead of inequality constraints, in the $\omega^{[7-9]}$ space it can be described as:

$$
\min \Phi(\omega, e)=\frac{1}{2} \omega^{\mathrm{T}} \boldsymbol{\omega}+\frac{\gamma}{2} \sum_{i=1}^{n} e_{i}^{2}
$$

In the formula, $\omega$ is the weight vector, $\mathrm{b}$ is parameters to be determined, $\gamma$ is tolerant of penalty coefficient, $e_{i}$ is the relaxation factor, the values are larger than 0.Constraint conditions are:

$$
y_{i}=\omega^{\mathrm{T}} \phi\left(x_{i}\right)+b+e_{i}
$$

To solve the above problems, the LSSVM regression function model is obtained:

$$
f(x)=\sum_{i=1}^{n} \alpha_{i} y_{i} K\left(x_{i}, x\right)+b
$$

\section{RESULT}

\section{A. Forecast Model Establishment}

According to the measured data of the horizontal displacement of deep foundation pit, a prediction model is established to predict the deformation of foundation pit support structure based on least square support vector machine. The edit program steps of subroutine in LSSVMlab toolbox as shown below: 1) Read data and standard samples; 2) LS-SVM parameter optimization: LS-SVM requires only two parameters to call. GAM and sig2 is the least squares support vector machine parameters, the GAM is the regularization parameter, determines the minimized and the degree of smoothing of Minimize and smooth of adaptive error, sig2 is the kernel function parameters, $\mathrm{B}$ is the threshold. Take NO19 point as the example, it is parameters result is $\mathrm{c}=0.0008, \mathrm{~g}=0.9859$. As shown in Figure 2 and $3 ; 3)$ Training the prediction model which has been determined to optimize the parameters: using trainlssvm function to build model, according to the input and output samples and preset training function parameters, the model train the network and get the support vector and response threshold of least squares support vector machine, the trainlssvm function is one of the important function of LSSVM toolbox ${ }^{[10,11]}$, is also the least squares support vector machine training function; 4) Model accuracy test: Call simlssvm function to predict the accuracy of the test, the function is similar to the SIM function in neural network toolbox, which was used to verify the accuracy of the model.

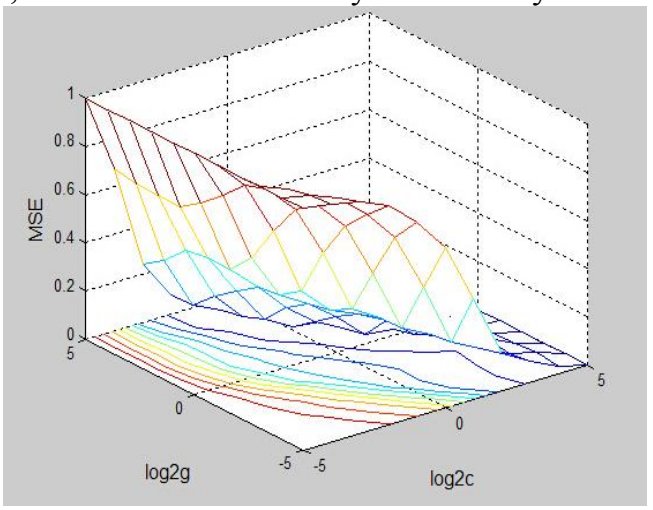

Figure 2. 3D View Plot of Parameter selections

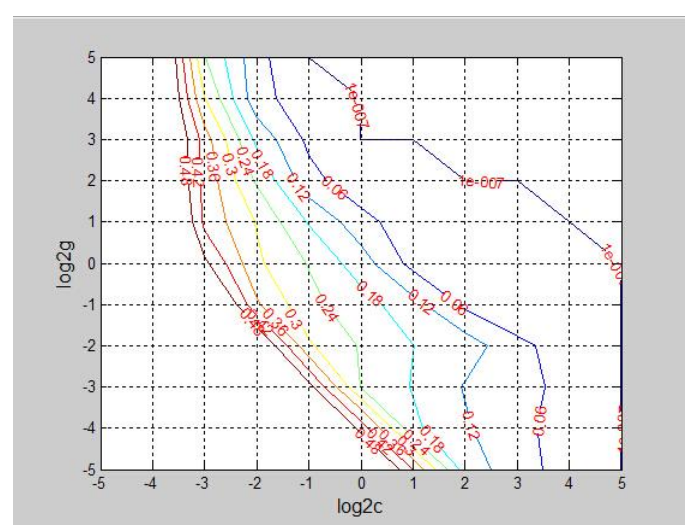

Figure 3. Contour map of Parameter selection 


\section{B. Prediction Accuracy Analysis}

The foundation pit horizontal displacement observation data has a total of 38 , so take the 28 observational data as the training samples of the least squares support vector machine (SVM) model, then predict the other 10 observation point displacement and be compared with the measured value to predict the accuracy. Due to the poor prediction result of some pretreated data. We selected NO3 and NO13 which the prediction result is better as the research object, and the prediction results and measured value were comparative analyzed, as shown in Figure 4, Figure 5.

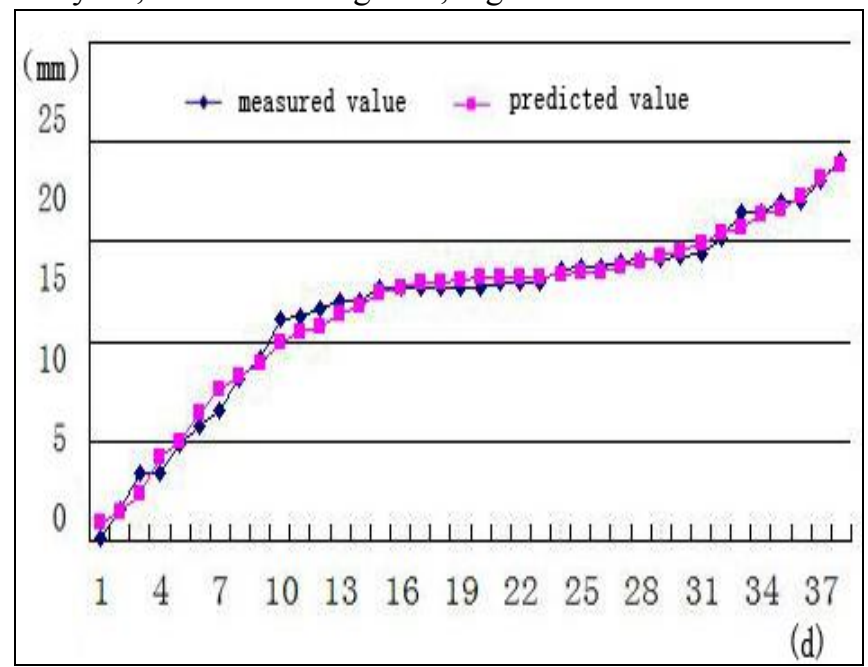

Figure 4. Contrast diagram between predicted value and measured value of NO3 observation point

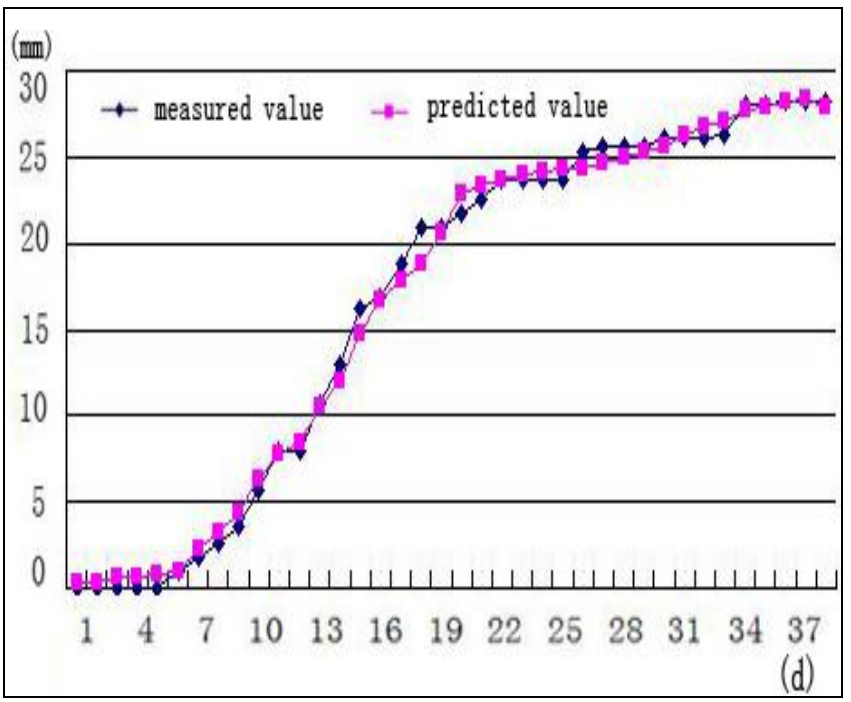

Figure 5. Contrast diagram between predicted value and measured value of NO13 observation point

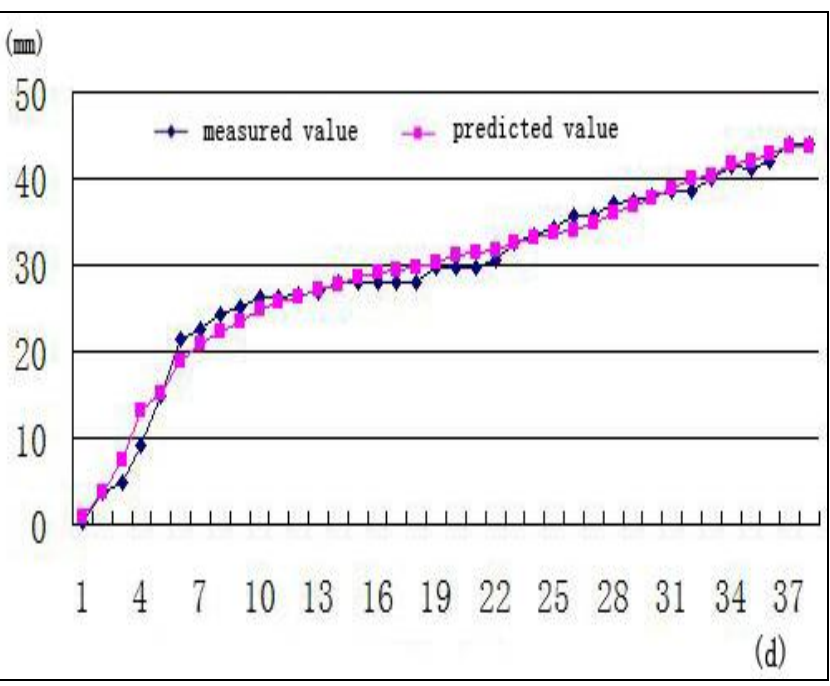

Figure 6. Contrast diagram between predicted value and measured value of $\mathrm{NO} 20$ observation point

The calculation result of relative mean error of each point as shown in table 1 , table 2 , and table 3 :

TABLE I. COMPARATIVE ANALYSIS TABLE BETWEEN PREDICTED VALUE AND MEASURED VALUE OF NO3 OBSERVATION POINT

\begin{tabular}{|c|c|c|c|}
\hline $\begin{array}{l}\text { Observation } \\
\text { time } \\
\text { series of NO3 }\end{array}$ & $\begin{array}{c}\text { Measured } \\
\text { Value } \\
(\mathbf{m m})\end{array}$ & $\begin{array}{c}\text { SVM } \\
\text { predicted } \\
\text { value } \\
(\mathbf{m m})\end{array}$ & $\begin{array}{c}\text { relative } \\
\text { error } \\
(\%)\end{array}$ \\
\hline 29 & 14.2 & 14.221 & 0.148 \\
\hline 30 & 14.3 & 14.506 & 1.441 \\
\hline 31 & 14.4 & 14.931 & 3.687 \\
\hline 32 & 15.2 & 15.400 & 1.316 \\
\hline 33 & 16.5 & 15.650 & 5.152 \\
\hline 34 & 16.5 & 16.305 & 1.182 \\
\hline 35 & 17.0 & 16.575 & 2.500 \\
\hline 36 & 17.0 & 17.253 & 1.488 \\
\hline 37 & 18.0 & 18.141 & 0.783 \\
\hline 38 & 19.1 & 18.911 & 0.989 \\
\hline \multicolumn{2}{|c|}{ average relative error $(\%)$} & \multicolumn{2}{|c|}{1.865} \\
\hline
\end{tabular}


TABLE II. COMPARATIVE ANALYSIS TABLE BETWEEN PREDICTED VALUE AND MEASURED VALUE OF NO13 OBSERVATION POINT

\begin{tabular}{|c|c|c|c|}
\hline $\begin{array}{c}\text { observation } \\
\text { time series } \\
\text { of NO13 }\end{array}$ & $\begin{array}{c}\text { measured } \\
\text { value(mm) }\end{array}$ & $\begin{array}{c}\text { SVM } \\
\text { predicted } \\
\text { value(mm) }\end{array}$ & $\begin{array}{c}\text { relative } \\
\text { error(\%) }\end{array}$ \\
\hline 29 & 25.6 & 25.307 & 1.144 \\
\hline 30 & 26.1 & 25.691 & 1.567 \\
\hline 31 & 26.1 & 26.251 & 0.578 \\
\hline 32 & 26.1 & 26.824 & 2.773 \\
\hline 33 & 26.3 & 27.099 & 3.038 \\
\hline 34 & 28.0 & 27.711 & 1.032 \\
\hline 35 & 28.0 & 27.909 & 0.325 \\
\hline 36 & 28.2 & 28.262 & 0.219 \\
\hline 37 & 28.2 & 28.358 & 0.560 \\
\hline 38 & 28.2 & 27.911 & 1.025 \\
\hline \multicolumn{2}{|c|}{ average relative } \\
error(\%) & & 1.226 \\
\hline
\end{tabular}

TABLE III. COMPARATIVE ANALYSIS TABLE BETWEEN PREDICTED VALUE AND MEASURED VALUE OF NO20 OBSERVATION POINT

\begin{tabular}{|c|c|c|c|}
\hline $\begin{array}{c}\text { observation } \\
\text { time series of } \\
\text { NO20 }\end{array}$ & $\begin{array}{c}\text { measured } \\
\text { value }(\mathbf{m m})\end{array}$ & $\begin{array}{c}\text { SVM } \\
\text { predicted } \\
\text { value }(\mathbf{m m}) \\
\end{array}$ & $\begin{array}{c}\text { relative } \\
\operatorname{error}(\%)\end{array}$ \\
\hline 29 & 37.3 & 36.778 & 1.399 \\
\hline 30 & 38.1 & 37.636 & 1.218 \\
\hline 31 & 38.5 & 38.784 & 0.738 \\
\hline 32 & 38.6 & 39.897 & 3.360 \\
\hline 33 & 40.1 & 40.428 & 0.818 \\
\hline 34 & 41.1 & 41.641 & 1.306 \\
\hline 35 & 41.1 & 42.068 & 2.355 \\
\hline 36 & 42.1 & 42.954 & 2.028 \\
\hline 37 & 44.1 & 43.679 & 0.955 \\
\hline 38 & 44.1 & 43.672 & 0.971 \\
\hline \multicolumn{2}{|c|}{ Average relative error $(\%)$} & \multicolumn{2}{|c|}{1.515} \\
\hline
\end{tabular}

\section{CONCLUSIONS}

The predicted and measured values are compared analysis and the result characteristic summarized as shown below:

- The predicted values predicted by the LS-SVM model are in agreement with the measured values, which are related to the input values of the training samples;

- The relative errors of the predicted values of the 3, 13 and 20 monitoring points were randomly changed, not according to a certain rule, which was caused by the instability of the deformation rate of the first 28 phase of the training samples;

- From the comparison chart of the predicted values and measured values of the selected two monitoring points can find that: the result of using LS-SVM prediction model for predicting the horizontal displacement of deep foundation pit can basically meet the requirement of deformation monitoring. It is feasible to apply the least squares support vector machine prediction model to predict deep foundation pit horizontal displacement, and the prediction results has certain practical significance to ensure the safety of foundation pit construction and early warning of danger.

\section{ACKNOWLEDGMENT}

This work was financially supported by the Program of Ministry of housing and Urban-Rural Development of the People's Republic of China (No. 2016-K5-019).

\section{REFERENCES}

[1] Xu Hongzhong,Yang Lei.Prediction foundation pit deformation based on least square upport vector machine regression[J].Journal of NanjingUniversity of Technology:Natural Science,2008,30(2):51-54.

[2] Li Wenli,Li Yuxia.Least square support vector machines model based on particle swarm optimization for hydrological forecasting[J].Journal of Computer Applications,2012,32(4):1 188-1 190.

[3] Peng Ling,Niu Ruiqing,Zhao Yannan,et al.Prediction of landslide displacement based on KPCA and PSO-SVR[J].Geomatics and Information Science of Wuhan University,2013,38(2):148-152

[4] Zhang Xuegong. Introduction to statistical learning theory and support vector machines .Acta Automatica Sinica, 2000, 26(1):32 42.

[5] Yu Ningfeng,Yang Huachao,Deng Kazhong,et al.Calculation of surface subsidence coefficient in mining areas using support vector machine regression.Journal of Liaoning Technical University: Natural Science, 2008,27(3):365-367.

[6] Yang Jiajia, Jiang Qigang, Chen Yongliang, et al. Lithology division for large-scale region segmentation based on LS-SVM and high resolution remote sensing images.Journal of China University of Petroleum: Natural Science,2012,36(1):60-67.

[7] Jiang Annan, Liang bing, Zhang Jiao. Forecasting in-situ gas content using geological factors based on particle swarm optimization and least square support vector machine.Journal of Liaoning Technical University: Natural Science,2009,28(3):363-366.

[8] Hsu C, Lin C.A comparison of methods for multiclass support vector machines. IEEE Transactions on Neural Networks, 2002, 13(2):415425.

[9] Eberhart R, Kennedy J.A new optimization using particle swarm theory//In proceedings of the 6th international symposium on micro machine and human science. Piscataway, NJ: IEEE Service Center, 1995:39-43.

[10] Li Wenli, Li Yuxia. Least square support vector machines model based on particle swarm optimization for hydrological forecasting. Journal of Computer Applications, 2012, 32(4):1 188-1 190.

[11] Eberhart R,Kennedy J.A new optimization using particle swarm theory//In proceedings of the 6th international symposium on micro machine and human science. Piscataway, NJ: IEEE Service Center, 1995:39-43. 